\title{
Competências e Habilidades Necessárias para um Ambiente Computacional em um Contexto de Transformação Digital
}

\author{
Tagiani B. D. de M. Goulart, Dantom G. Helfer, Sandra E. L. Iepsen, Liane M. \\ Kipper, Rejane Frozza
}

\author{
Programa de Pós-Graduação em Sistemas e Processos Industriais (PPGSPI) - \\ Universidade de Santa Cruz do Sul (UNISC) \\ Santa Cruz do Sul - RS - Brazil \\ tagiani@mx2.unisc.br, dantomh@gmail.com, seliepsen@gmail.com, \\ liane@unisc.br, frozza@unisc.br
}

\begin{abstract}
The aim of this study was to identify and understand the view of undergraduate students on the competencies and skills necessary for the development of computational environments, through digital transformation. Theoretical studies on the research topic were carried out. A questionnaire was also applied to students of the Computer Science course at a university in Rio Grande do Sul. From the results found, it is highlighted that students recognize the "communication" competence as being essential for teamwork. Collective and continuous learning, as well as environments that promote the generation and discussion of ideas, also stood out.
\end{abstract}

Resumo. O objetivo deste estudo foi identificar e compreender a visão de acadêmicos de curso de graduação sobre as competências e habilidades necessárias para o desenvolvimento de ambientes computacionais, mediante a transformação digital. Estudos teóricos sobre o tema de pesquisa foram realizados. Também foi aplicado um questionário junto a estudantes de cursos da área de Computação em uma universidade gaúcha. Dos resultados encontrados destaca-se que os estudantes reconhecem a competência "comunicação" como sendo essencial para trabalho em equipe. O aprendizado coletivo e contínuo, assim como ambientes que promovam a geração e discussão de ideias também se destacam.

\section{Introdução}

Passamos por uma revolução tecnológica, a qual está em estágio emergente, produzindo novos conceitos e definições, os quais devem ser compreendidos. Nesta perspectiva, é fundamental que a educação se recrie em meio ao contexto baseado no compartilhamento imediato de informações. Novas formas de ensinar e aprender devem ser pensadas, as quais atendam as demandas de nível micro e macrossocial. Em paralelo, a formação de trabalhadores deve estar voltada para o desenvolvimento de competências interdisciplinares, com foco na ampliação de capacidades para resolução de problemas.

Desta forma, as organizações passam a reestruturar a dinâmica do trabalho e suas condições de execução, no qual a tecnologia é direcionada como possibilidade para resolução de problemas (Leduc e Ponge, 2018). Via de regra, o presente movimento dá origem a produtos inteligente e conectados, trazendo também desafios em diferentes 
níveis (Sánchez, 2017), fomentando a ascensão de um novo modelo de negócio. O impacto desta revolução reflete nas profissões e no mercado de trabalho, logo, o desenvolvimento de competências interpessoais e relacionais é imprescindível. Desta forma, surgem novas demandas, relacionadas à produção de conhecimento e desenvolvimento de habilidades dos colaboradores (Freddi, 2018).

Van Dalen, Henkens e Schippers (2010) descreven duas categorias de competências, sendo elas: hard skills e soft skills. As hard skills estão relacionadas à capacidade adquirida através do estudo teórico ou prático, podendo ser relacionada com capacidade mental e física de lidar com a carga de trabalho. Já as soft skills referem-se às competências comportamentais e pessoais, englobando as habilidades sociais de confiabilidade e compromisso.

A presente pesquisa foi realizada no ano de 2019 junto aos acadêmicos das disciplinas de Inteligência Artificial Avançada e Pesquisa Aplicada dos cursos de Ciência da Computação e Engenharia de Computação e Inteligência Artificial Aplicada à Educação do curso de Computação Licenciatura de uma Universidade situada no Rio Grande do Sul. O objetivo foi identificar e compreender a visão dos acadêmicos de cursos de Computação sobre as competências e habilidades necessárias para o desenvolvimento de um ambiente computacional.

O artigo está organizado nas seguintes seções: a seção 2 apresenta a Fundamentacao Teórica dos temas relacionados à pesquisa; a seção 3 descreve a metodologia seguida; a seção 4 apresenta os resultados e discussões; na seção 5 são apresentadas as conclusões da pesquisa.

\section{Fundamentação Teórica}

Comparando-se o modelo conceitual de habilidades e competências com os modelos contemporâneos da inteligência humana, pela ótica da psicometria e psicologia cognitiva, há indicação de que os modelos se referem a dimensões comuns da capacidade humana (Primi et al., 2001). Brandão e Borges-Andrade (2007) indicam que a noção de competência se caracteriza complexa e multifacetada, podendo ser interpretada de diferentes maneiras. Fleury e Fleury (2004) comentam que as competências se manifestam pelos sujeitos e estão relacionadas com seu empenho na realização de tarefas, sendo possível diferenciá-las de duas formas:

i) foco nas aptidões, levando em consideração os talentos naturais de cada indivíduo e como eles são aprimorados; e

ii) conhecimento, ou seja, habilidades que o sujeito precisa desenvolver para desempenhar determinada tarefa.

Competências são caracterizadas por diversas áreas. Dentre elas destacam-se as competências técnicas, as quais são descritas a partir dos conhecimentos específicos sobre a tarefa a ser realizada. As competências acerca dos processos, que se baseiam através dos conhecimentos a partir do processo de trabalho. E as competências sociais, incluindo o "saber ser", que está interligada à interação social dos indivíduos (Fleury e Fleury, 2004).

Já os ambientes virtuais de aprendizagem são sistemas computacionais acessíveis aos usuários, os quais dispõem de mecanismos de interação direcionados ao 
processo de aprendizado (Frozza et al., 2011). Ribeiro e Moreira (2014) comentam que o sucesso da educação em ambientes online depende não só das condições tecnológicas e sociais mas, também, das condições pedagógicas. Bogo e Martins (2003) revelam que, o uso de agentes inteligentes facilitou a autonomia, comunicabilidade, cooperatividade, promovendo condições pedagógicas na proposta desenvolvida pelos autores, com a formação de um profissional para além das fortes habilidades técnicas e matemáticas. Os autores comentam o uso de novas tecnologias educacionais, como a hipermídia e o ensino a distância, como elementos de uma ecologia cognitiva igualmente necessária e estimulante na formação do engenheiro do século XXI (Timm, Schnaid, Amoretti, 2003).

Mas aspectos como a estrutura organizacional e o fator humano podem afetar drasticamente a qualidade de softwares devido à falta de coordenação entre equipes, falta de confiança e falha na comunicação (Herbsleb e Grinter, 1999; Suali et al., 2017). Nesta mesma perspectiva, Bird (2011) demonstra que a qualidade de um software está intimamente relacionada com o alto nível de responsabilidade do desenvolvedor. Além disso, estudos empíricos comprovam que a qualidade e o número de falhas póslançamento estão relacionados com a fragmentação das contribuições entre os desenvolvedores. Neste sentido, é necessário que a equipe mantenha o foco nos objetivos definidos, pois a divisão de contribuições pode afetar a qualidade e eficiência do software (Pinzger, Nagappan e Murphy, 2011).

Khan et al. (2017) indicam 22 aspectos considerados como barreiras relacionadas ao processo de melhorias de softwares, sendo eles: profissionais inexperientes; falta de recursos; rotatividade de pessoal; falta ou falha de comunicação; diferenças culturais; falta de apoio organizacional; falta de confiança; distância temporal; falta de conhecimento de melhoria de processos; infraestrutura organizacional deficiente; falta de treinamento; restrição orçamentária; falta de feedback; mudanças organizacionais; conflitos de personalidade; falta de patrocínio; carga de trabalho; falta de metodologia formal de implementação do Software Process Improvement (SPI); política organizacional; falta de ferramentas e padrões de implementação; pressão do tempo e parar na implementação do plano de ação.

As competências comportamentais têm representado grande diferencial para o sucesso e fracasso de uma carreira pública ou não e de um negócio próprio (De Souza Cruz e De Moraes, 2013; Capuano, 2015). De Souza Cruz e De Moraes (2013) realizaram um estudo com empreendedores paulistas e indicam que a resiliência é um conhecimento comportamental importante para alcançar o sucesso. Capuano (2015) estudou experiências dos países da Organização para a Cooperação e Desenvolvimento Econômico (OECD) na adoção de modelos de gestão por competências para o setor público e comenta que, mesmo existindo resistências a este tipo de gestão, conclui que "...a gestão por competências constitui um verdadeiro "estilo de vida" de uma organização que planeja sua força de trabalho, recruta e seleciona talentos, avalia o desempenho individual, reconhece, promove, remunera e substitui seus executivos, gerentes e colaboradores com base em competências". 


\section{Metodologia}

A pesquisa foi de âmbito qualitativo, para que se possa avaliar as respostas obtidas de forma qualificada. "Na pesquisa qualitativa, diferentemente da quantitativa, o pesquisador busca compreender os fenômenos observando-os, interpretando-os e descrevendo-os" (Mello et al., 2012, p. 1). A abordagem foi exploratória, realizando um estudo de campo, pois buscou coletar e analisar a opinião dos acadêmicos das disciplinas de Inteligência Artificial Avançada e Pesquisa Aplicada dos cursos de Ciência da Computação e Engenharia de Computação e Inteligência Artificial Aplicada à Educação do curso de Computação Licenciatura de uma universidade situada no Rio Grande do Sul, com relação às competências e habilidades necessárias para o desenvolvimento de um ambiente computacional.

A fim de alcançar o objetivo, estudos teóricos foram realizados sobre o tema. Para coletar os dados do grupo correspondente, um questionário foi elaborado. $\mathrm{O}$ questionário é um instrumento desenvolvido cientificamente, composto por um conjunto de perguntas ordenadas (Marconi et al., 2002; Gil, 2002) e que, no campo organizacional, é utilizado para obter informações sobre empresas e indivíduos (Hair et al., 2005). As questões utilizadas na pesquisa são descritas nos resultados e discussões.

Foi realizada à Análise do Conteúdo, proposta por Bardin (2011), que tem como objetivo analisar de forma crítica os conteúdos obtidos. Esta abordagem pode ser utilizada nas pesquisas qualitativas e quantitativas, pois permite ao pesquisador interpretar de forma subjetiva, relacionando com aspectos teóricos.

Tabela 1. Polos de Comunicação

\begin{tabular}{|c|c|c|c|}
\hline Emissor & Receptor & Mensagem & Canal \\
\hline $\begin{array}{l}\text { Executa } \\
\text { mensagem } \\
\text { que pode ser } \\
\text { aplicada de } \\
\text { forma } \\
\text { individual ou } \\
\text { grupal. }\end{array}$ & $\begin{array}{l}\text { Recebe a mensagem de } \\
\text { forma individual ou } \\
\text { grupal. } \\
\text { Estuda sobre o que a } \\
\text { mensagem se destina. }\end{array}$ & $\begin{array}{l}\text { É o ponto de partida } \\
\text { da análise. } \\
\text { O conteúdo é } \\
\text { interpretado pelo } \\
\text { receptor. }\end{array}$ & $\begin{array}{lr}\text { Serve } & \text { como } \\
\text { procedimento } & \\
\text { experimental } & \text { de } \\
\text { conteúdo. } & \end{array}$ \\
\hline
\end{tabular}

Os polos de comunicação (Tabela 1) são ferramentas que possibilitam ao pesquisador encontrar as semelhanças nos resultados obtidos, independente da área de atuação. Para interpretação das respostas, o referencial teórico deve estar interligado com os resultados encontrados, para que haja sentido à interpretação (Bardin, 2011).

\section{Resultados e Discussões}

A pesquisa realizada nas disciplinas de Inteligência Artificial Avançada e Pesquisa Aplicada dos cursos de Ciência da Computação e Engenharia de Computação, Inteligência Artificial Aplicada à Educação do curso de Computação Licenciatura, contou com uma amostra de treze estudantes. $\mathrm{O}$ tamanho da amostra foi reduzido, pois a 
pesquisa foi proposta para ser respondida de forma voluntária e o total de estudantes destas disciplinas, no momento da pesquisa, contava com 40 estudantes.

A faixa etária dos respondentes foi de 21 a 32 anos, sendo o gênero predominante o masculino. Sendo que as respostas predominantes são dos acadêmicos de Ciência da Computação (69,20\%), Engenharia da Computação (23,10\%) e Computação Licenciatura $(7,70 \%)$.

Quanto às perguntas diretivas, obteve-se os seguintes resultados:

Pergunta 1: "Uma equipe de desenvolvimento de sistema requer habilidades. Das habilidades descritas a seguir indique duas mais importantes no seu ponto de vista e justifique por que as escolhidas são as mais importantes".

Destacou-se como predominantes o conhecimento em desenvolvimento de sistema (hard skill) com 61,54\% e comunicação (soft skill) com 53,85\%, conforme ilustra a Figura 1.

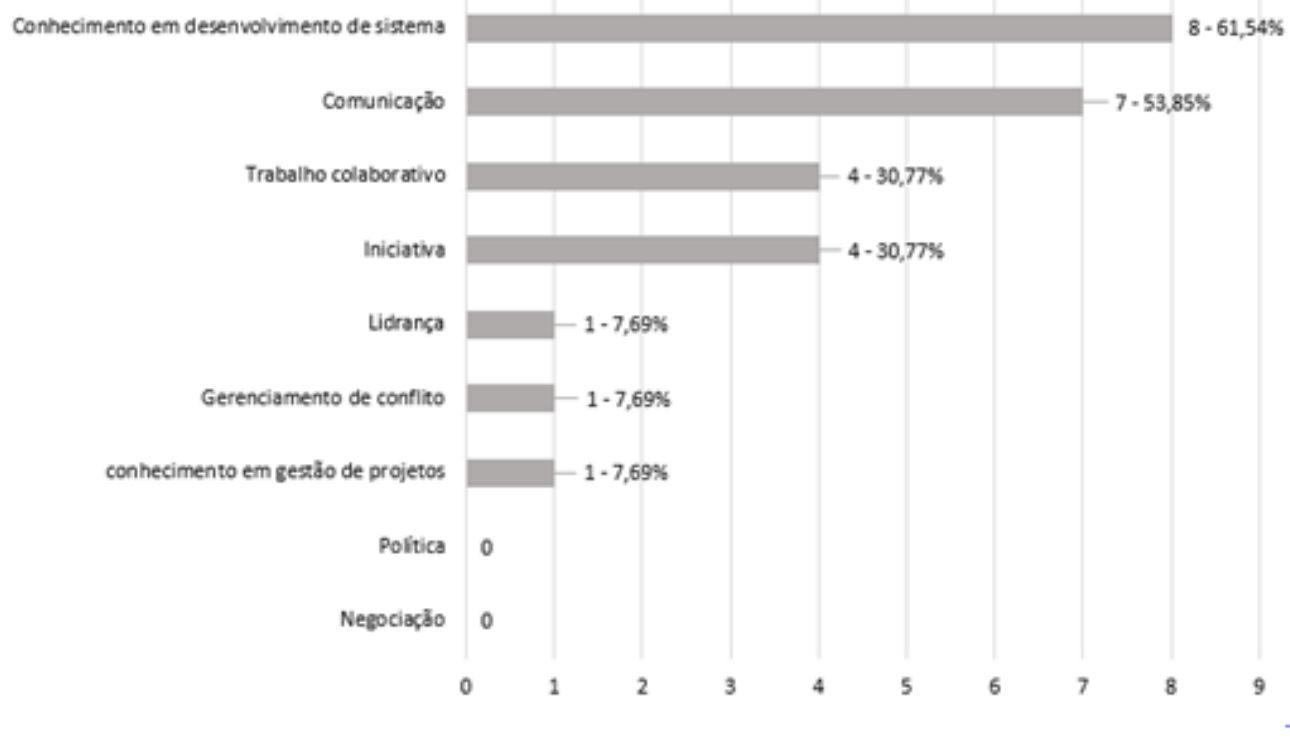

Figura 1 - Habilidades para equipe de desenvolvimento de sistemas

Gramigna (2007) e Santos (2018) destacam que a comunicação está ligada à forma que as pessoas se relacionam, criando uma capacidade de escuta e compreensão das demandas trazidas ou geradas. Destaca-se ainda que ter boa comunicação torna-se essencial, pois facilita o relacionamento entre os membros do grupo, auxiliando na resolução de problemas e no desenvolvimento interpessoal. Para uma equipe de desenvolvedores não é diferente, pois a lógica da programação está interligada com a razão e a comunicação, uma vez que trabalha com diversas linguagens e estruturas conhecidas (Neto et al., 2018). Nas percepções dos estudantes "uma boa comunicação auxilia na redução de gaps entre os desenvolvedores, evitando falhas e retrabalhos desnecessários, bem como auxilia na resolução de problemas, aumentando a eficiência da equipe." O conhecimento técnico também foi amplamente abordado pelos estudantes, pois ele é necessário para o desenvolvimento de sistemas, como fundamento básico do saber fazer. 
Pergunta 2: "O ambiente de trabalho de uma equipe de desenvolvimento de sistema necessita promover competências. Indique as duas mais importantes no seu ponto de visto e justifique." $\mathrm{Na}$ percepção dos estudantes estar aberto ao aprendizado $(61,54 \%)$ e à geração e discussão de ideias (46,15\%) são as principais competências para um desenvolvedor, conforme evidenciado na Figura 2.

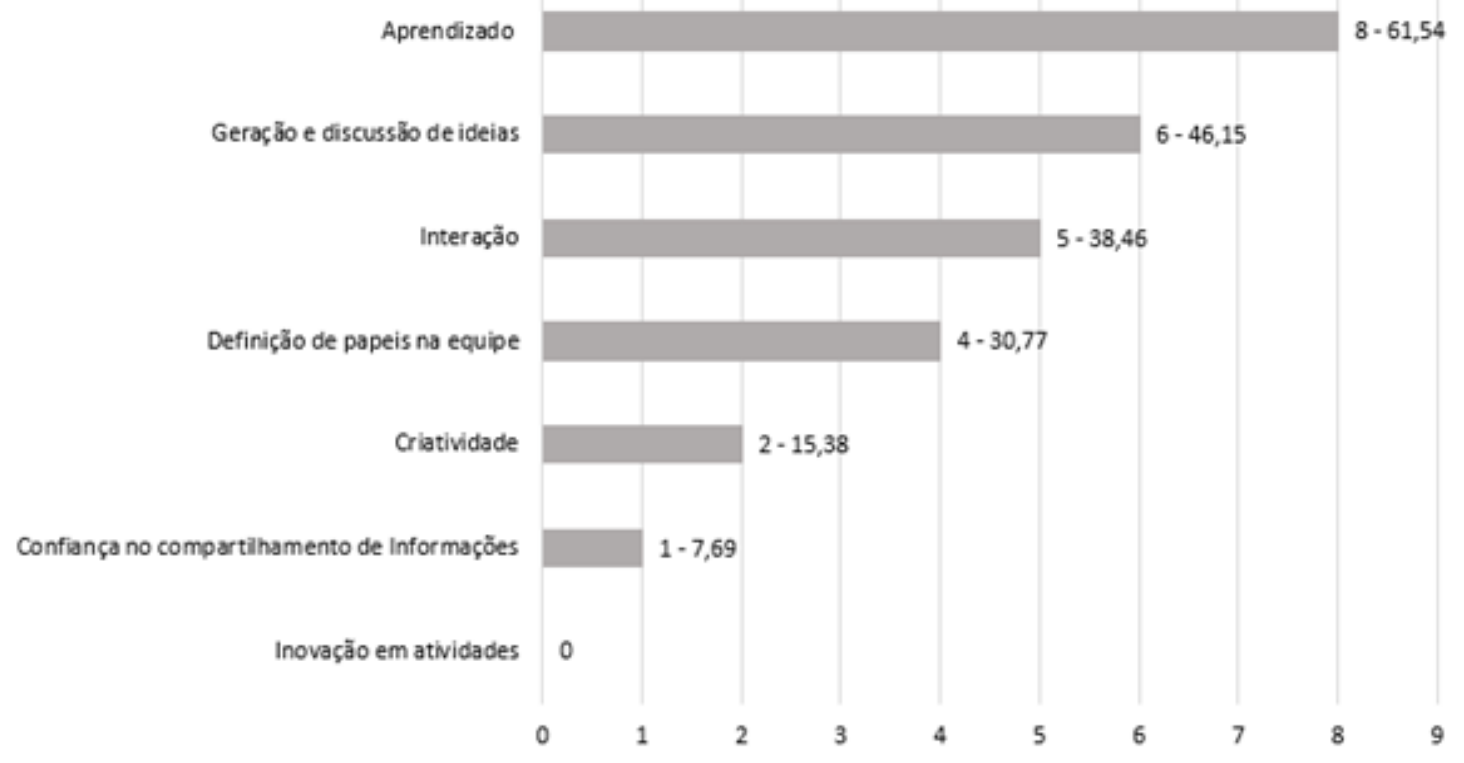

Figura 2 - Competências para equipe de desenvolvimento de software.

Conforme mencionado pelos estudantes observa-se a necessidade de um ambiente de constante aprendizado, pois novas tecnologias surgem a todo momento, permitindo que membros da equipe evoluam. O aprendizado coletivo instiga os profissionais a inovar, não se satisfazendo com os mesmos recursos e os mesmos métodos de desenvolvimento, e buscar melhores qualificações. As trocas de conhecimento tornam a equipe mais forte.

A geração e discussão de ideias é essencial para melhoria contínua e criação de produtos e projetos, ou seja, estimula a criatividade, neste context, é importante ter uma equipe organizada. Em conjunto, a geração e discussão de ideias e o aprendizado são considerados os principais benefícios de se trabalhar em equipe, além de facilitar o surgimento de novos projetos.

Pergunta 3: "Se você tivesse a tarefa de desenvolver um sistema, quais ações seriam importantes para a promoção das habilidades e competências definidas por você como as mais importantes nas questões anteriores?"

Quando questionados sobre as ações importantes para desenvolver habilidades e competências, enfatizamos as seguintes sugestões:

- Reunir a equipe periodicamente e definir os papeis de cada membro da equipe, de acordo com os conhecimentos em desenvolvimento de sistema que cada um possui. Seguindo isso discutir junto com a equipe qual seria a melhor forma de realizar a tarefa solicitada, de forma que todos possam interagir e compartilhar seus conhecimentos. 
- Ter um gerenciador de processos, ou seja, um bom canal de comunicação e fazer o reconhecimento dos membros da equipe por suas capacidades.

- Discussão sobre ferramentas e conceitos atuais e delimitar tempo para pesquisa.

- Proporcionar momentos de integração para fortalecer a união da equipe e motivá-la a melhorar.

- Identificar cada etapa do projeto: início, organização, execução e encerramento.

- Ter uma boa comunicação e negociação, para poder entender o que realmente o cliente está precisando.

\section{Conclusão}

Comparando as soft skills com as hard skills, as respostas dos estudantes revelam que é interessante haver equilíbrio entre conhecimento no desenvolvimento de sistema, boa comunicação e trabalho colaborativo em equipes que desenvolvem ambientes computacionais. O desenvolvimento de ambientes colaborativos, tanto para a discussão de problemas e ideias, como também de mentoria e troca de conhecimento também foi indicado como um caminho para o compartilhamento rápido e imediato de informações. A capacidade de aprendizado, tanto individual como em grupo e até organizacional, promove a geração de conhecimento, levando a melhorias de processos e de interação nas equipes de desenvolvedores de sistemas. Os resultados revelam que no contexto da transformação digital as tecnologias são importantes, mas para a criação de novas tecnologias e soluções há necessidade de colaboração e cocriação humana.

Como sugestão de trabalhos futuros, novos estudos deverão ser realizados, contando com a presença de estudantes de outros cursos de graduação, como, por exemplo, a engenharia de produção, buscando comparar os resultados.

\section{Agradecimentos}

O presente trabalho foi realizado com apoio da Coordenação de Aperfeiçoamento de Pessoal de Nível Superior - Brasil (CAPES) - Código de Financiamento 001, da FAPERGS, , National Council for Scientific and Technological Development (CNPq), Brazil (process number 303934/2019-0) e da UNISC por meio do programa de bolsas PUIC.

\section{Referências}

BARDIN, Lawrence. Análise de conteúdo. Lisboa: edições, v. 70, p. 225, 2011.

BIRD, Christian et al. Don't touch my code!: examining the effects of ownership on software quality. In: Proceedings of the 19th ACM SIGSOFT symposium and the 13th European conference on Foundations of software engineering. ACM, 2011. p. 414.

BOGO, Luis Henrique; MARTINS, Alejandro. Formação de Comunidades Virtuais em Ambientes de E-learning: uma Aplicação Utilizando Agentes Inteligentes. Informática na Educação: teoria \& prática, Porto Alegre, v. 6, n. 2, p. 11-20, jul./dez. 2003. 
BRANDÃO, Hugo Pena; BORGES-ANDRADE, Jairo Eduardo. Causas e efeitos da expressão de competências no trabalho: para entender melhor a noção de competência. RAM. Revista de Administração Mackenzie, v. 8, n. 3, p. 32-49, 2007.

CAPUANO, Ethel Airton. Gestão por competências no setor público: experiências de países avançados e lições para o Brasil. Revista do Serviço Público, v. 66, n. 3, p. 371-394, 2015. CORREIA, Wilson. Os diversos tipos de conhecimento. Acesso em, v. 4, n. 08, p. 2009, 2006.

DE SOUZA CRUZ, Myrt Thânia; DE MORAES, Isabel Mingotti Machado. EMPREENDEDORISMO E RESILIÊNCIA: mapeamento das competências técnicas e comportamentais exigidas na atualidade. Pensamento \& Realidade, v. 28, n. 2, 2013.

FLEURY, Maria Tereza Leme; FLEURY, Afonso Carlos Correa. Alinhando estratégia e competências. Revista de administração de empresas, v. 44, n. 1, p. 44-57, 2004.

FREDDI, Daniela. Digitalisation and employment in manufacturing. AI \& SOCIETY, v. 33, n. 3, p. 393-403, 2018.

FROZZA, R., DA SILVA, A. A. K., SCHREIBER, J. N. C., LUX, B., MOLZ, K. W., KIPPER, L. M., BORIN, M. P., DE CARVALHO, A. B., BAIERLE, J. L., AND SAMPAIO, L. Agentes pedagógicos emocionais atuando em um ambiente virtual de aprendizagem. RENOTE, v.9, n. 1. 2011.

GIL, Antônio Carlos. Como classificar as pesquisas. Como elaborar projetos de pesquisa, v. 4, p. 44-45, 2002.

GRAMIGNA, Maria Rita. Modelo de competências e gestão dos talentos. In: Modelo de competências e gestão dos talentos. 2007.

HAIR, Joseph et al. Fundamentos de métodos de pesquisa em administração. Bookman Companhia Ed, 2005.

HERBSLEB, James D.; GRINTER, Rebecca E. Splitting the organization and integrating the code: Conway's law revisited. In: Proceedings of the 21 st international conference on Software engineering. ACM, 1999. p. 85-95.

KHAN, Arif Ali et al. Systematic literature review and empirical investigation of barriers to process improvement in global software development: Client-vendor perspective. Information and Software Technology, v. 87, p. 180-205, 2017.

LEDUC, SYLVAIN; PONGE, LUDOVIC. La evolución digital y los cambios organizativos: qué respuestas de la ergonomía? Laboreal, v. XIV, n. 2, p. $31-44$, 2018 .

MARCONI, Marina de Andrade et al. Técnicas de pesquisa. São Paulo: Atlas, 2002.

MELLO, Carlos Henrique Pereira et al. Action research in production engineering: a structure proposal for its conduction. Production, v. 22, n. 1, p. 1-13, 2012.

NETO, Manassés et al. Robótica educacional uma ferramenta para ensino de lógica de programação no ensino fundamental. In: Anais do Workshop de Informática na Escola. 2018. p. 315. 
PRIMI, Ricardo et al. Competências e habilidades cognitivas: diferentes definições dos mesmos construtos. Psicologia: teoria e pesquisa, v. 17, n. 2, p. 151-159, 2001.

PINZGER, Martin; NAGAPPAN, Nachiappan; MURPHY, Brendan. Can developermodule networks predict failures? In: Proceedings of the 16th ACM SIGSOFT International Symposium on Foundations of software engineering. ACM, 2008. p. 212 .

RIBEIRO, Glória; MOREIRA, J. António. Percepção de competências de aprendizagem em ambientes online no ensino da Educação Física. Informática e Educação: Teoria \& Prática, v. 17, n. 1, p. 155-168, 2014.

SÁNCHEZ, Marisa. A framework to assess organizational readiness for digital transformation. Dimensión Empresarial, v. 15, n. 2, p. 27-40, 2017.

SANTOS, Rosineia Oliveira. A importância da comunicação no processo de liderança. Revista de Administração em Saúde, v. 18, n. 72, 2018.

SUALI, A. J. et al. Developers' coordination issues and its impact on software quality: A systematic review. In: Science in Information Technology (ICSITech), 2017 3rd International Conference on. IEEE, 2017. p. 659-663.

TIMM, Maria Isabel; SCHNAID, Fernando; AMORETTI, Maria Suzana Marc. Elaboração de Projetos, Hipermídia e E-learning-by-doing: ferramentas pedagógicas para o ensino de engenharia. Informática na educação: teoria \& prática. Porto Alegre. Vol. 6, n. 2 (jul./dez. 2003), p. 39-57, 2003.

VAN DALEN, Hendrik P.; HENKENS, Kène; SCHIPPERS, Joop. Productivity of older workers: Perceptions of employers and employees. Population and development review, v. 36, n. 2, p. 309-330, 2010. 